Revue de droit comparé du travail et de la sécurité sociale

$1 \mid 2021$

Doctrine

\title{
Le droit du travail confronté à la crise du Covid-19
}

\section{Nouri Mzid}

\section{OpenEdition}

Journals

Édition électronique

URL : https://journals.openedition.org/rdctss/709

DOI : $10.4000 /$ rdctss.709

ISSN : 2262-9815

\section{Éditeur}

Centre de droit comparé du travail et de la sécurité sociale

\section{Édition imprimée}

Date de publication : 1 avril 2021

Pagination : 134-137

ISSN : 2117-4350

\section{Référence électronique}

Nouri Mzid, «Le droit du travail confronté à la crise du Covid-19», Revue de droit comparé du travail et de la sécurité sociale [En ligne], 1 | 2021, mis en ligne le 01 avril 2021, consulté le 01 avril 2022. URL : http://journals.openedition.org/rdctss/709 ; DOI : https://doi.org/10.4000/rdctss.709

\section{(c) (i) (9)}

Revue de droit comparé du travail et de la sécurité sociale est mise à disposition selon les termes de la Licence Creative Commons Attribution - Pas d'Utilisation Commerciale - Pas de Modification 4.0 International. 


\section{NOURI MZID}

UNIVERSITÉ DE SFAX

\section{LE DROIT DU TRAVAIL CONFRONTÉ À LA CRISE DU COVID-19}

En Tunisie, comme partout ailleurs, la pandémie de Covid-19 a engendré une crise inédite, ayant créé un climat d'insécurité totale sur le plan sanitaire et un grand bouleversement du paysage socio-économique. Pour faire face à cette situation douloureuse, les pouvoirs publics ont été amenés à intervenir par des textes juridiques de circonstances ${ }^{1}$, en adoptant des mesures exceptionnelles qui ont touché les domaines les plus variés.

En matière de droit du travail, plusieurs dispositions dérogatoires ont été adoptées visant à assurer une certaine préservation de l'emploi face à la crise du Covid-19, et une prise en considération de l'impact de cette crise sur l'exécution du travail.

\section{I - LA PRÉSERVATION DE L'EMPLOI FACE À LA CRISE DU COVID-19}

La préservation de l'emploi a été l'un des soucis majeurs des pouvoirs publics pour faire face aux implications de la crise du Covid-19, qui s'est traduite notamment par des fermetures d'entreprises et de nombreuses pertes d'emplois².

À cet égard, le législateur a utilisé la technique de l'hibernation des dispositions du Code du travail relatives à l'effet de la force majeure sur le sort du contrat de travail. En effet, aux termes de l'article 14, al. 3 (c) du Code du travail, le contrat de travail prend fin «en cas d'empêchement d'exécution résultant d'un cas fortuit ou de force majeure, survenu avant ou pendant l'exécution du contrat ». Or, les mesures restrictives décrétées par les pouvoirs publics pour faire face à la propagation du coronavirus, notamment l'arrêt des activités par suite du confinement général ${ }^{3}$, l'interdiction des déplacements entre les villes et le couvre-feu constituent naturellement un cas de force majeure en raison de leur caractère imprévisible, insurmontable et extérieur à la volonté de l'employeur, risquant dès lors d'entraîner la rupture du contrat de travail en cas d'application des dispositions de l'article 14, al. 3 (c) du Code du travail. Dans un souci d'assurer la protection de l'emploi, le législateur a ainsi suspendu l'application de ces dispositions durant toute la période

1 À cet égard, le Chef du Gouvernement a été habilité par la loi n²020-19 du 12 avril 2020 à légiférer par décret-loi dans l'objectif de faire face aux répercussions de la propagation du Coronavirus.

2 Selon une enquête réalisée par l'Institut national de la statistique, en collaboration avec International Finance Corporation, sur l'impact de la crise Covid-19 sur le secteur privé (décembre 2020), le taux des fermetures définitives d'entreprises a atteint $5,4 \%$ au $3^{\text {ème }}$ trimestre 2020 , contre $0,4 \%$ au $2^{\text {ème }}$ trimestre de la même année. Selon la même enquête menée au mois de juillet 2020, 59\% des entreprises ont déclaré avoir procédé à des ajustements liés à l'emploi, contre 53,7\% en avril de la même année. Par ailleurs, le taux des entreprises ayant procédé à des licenciements a atteint $17,7 \%$ en juillet, contre $8 \%$ en avril.

3 La Tunisie a connu deux phases de confinement général. La première phase a débuté le 22 mars 2020 et sa levée a été décidée le 3 juillet de la même année. La deuxième phase a été annoncée pour une plus courte durée, du 14 au 17 janvier 2021. 
de confinement général ${ }^{4}$, rendant de ce fait inopérante la force majeure comme motif de rupture du contrat de travail.

La suspension de l'effet de la force majeure en droit du travail a été étendue aussi aux dispositions de l'article 21-12 du Code du travail relatif à la procédure de contrôle des licenciements et de mise en chômage économiques. Aux termes de cet article, "sont abusifs le licenciement ou la mise en chômage intervenus sans l'avis préalable de la commission régionale ou de la commission centrale de contrôle du licenciement, sauf cas de force majeure ". La suspension de l'application de ces dispositions ${ }^{5}$ a ainsi rendu inopérante la force majeure en tant que motif permettant de recourir au licenciement ou à la mise en chômage économique, sans passer par la procédure de contrôle administratif exigée par la loi en la matière.

Néanmoins, la suspension des dispositions du Code du travail relatives à l'effet de la force majeure sur le sort du contrat de travail revêt un caractère exceptionnel et provisoire, et demeure alors une solution insuffisante, étant limitée à la durée du confinement général. Du reste, aucune mesure n'a été adoptée en vue d'interdire explicitement les licenciements ou mises en chômage décidés en raison du confinement général décrété par les pouvoirs publics.

En revanche, quelques mesures d'accompagnement ont été adoptées en faveur des entreprises et des salariés affectés par l'interruption de leur activité à cause de la crise du Covid-19. Ainsi, des indemnités exceptionnelles et provisoires ont été attribuées aux salariés concernés, au titre des périodes d'interruption partielle ou totale de l'activité de l'entreprise, tout en maintenant au profit de ces salariés le bénéfice des prestations de soins au sein des établissements publics de santé, ainsi que le bénéfice des prestations sociales, durant la période d'interruption de l'activitéb. De plus, les entreprises lésées ont bénéficié d'un report de paiement des cotisations patronales dans le régime légal de sécurité sociale, au titre du deuxième trimestre de l'année 2020, et ce pour trois mois, sans application de pénalités de retard au titre dudit report ${ }^{7}$.

Conformément au décret-loi n²020-4 du 14 avril 2020, I'octroi des indemnités aux salariés affectés par la crise du Covid-19 était initialement prévu pour la seule période de confinement total, décrétée le 22 mars 2020 et prolongée jusqu'au 3 juillet 2020. Mais le décret-loi n²020-32 du 10 juin 2020, sans avoir abrogé celui du 14 avril 2020, est également venu prévoir l'octroi d'indemnités exceptionnelles complémentaires, au titre des périodes postérieures, en faveur des salariés appartenant à certaines catégories d'entreprises lésées dont la liste a été fixée par un arrêté du Ministre des affaires sociales en date du

4 Art. $1^{\text {er }}$ du décret-loi du Chef du Gouvernement n²020-2 du 14 février 2020 portant suspension exceptionnelle et provisoire de certaines dispositions du Code du travail.

5 Art. 2 du décret-loi n²020-2 du 14 février 2020.

6 Voir les décrets-lois du Chef du Gouvernement n²020-4 du 14 février 2020, et n²020-32 du 10 juin 2020.

7 Art. 14 du décret-loi du Chef du Gouvernement n²020-4 du 14 février 2020.

8 Aux termes de l'article 10 du décret-loi n²020-32 du 10 juin 2020, « les dispositions du présent décret-loi s'appliquent aux entreprises lésées et à leurs salariés mentionnés à ses articles 2 et 3 , et ce, sans préjudice des dispositions du décret-loi du Chef du Gouvernement n 2020-4 du 14 avril $2020 »$. 
20 juillet 2020. Ainsi, dans ce cadre, il a été décidé d'accorder aux salariés des entreprises du tourisme et de l'artisanat, ayant cessé leur activité à cause de la crise du Covid-19, des indemnités exceptionnelles complémentaires pendant la période de suspension d'activité, pour une durée pouvant aller jusqu'à 6 mois tout au long de l'année $2021^{10}$.

Les indemnités accordées aux salariés affectés par la crise du Covid-19 ne revêtent certainement pas le caractère d'un salaire, ni celui d'une assurance-chômage, dont le régime est encore inconnu en droit tunisien ${ }^{11}$. II s'agit plutôt de garantir aux salariés, empêchés de travailler pour cause de confinement, des moyens de subsistance, sous la forme d'une aide sociale exceptionnelle accordée par les pouvoirs publics et dont le montant reste très faible ${ }^{12}$.

Les mesures sociales d'accompagnement indiquées ont été soumises à plusieurs conditions, dont notamment l'existence d'une interruption ou baisse d'activité ou de difficultés financières subies par l'entreprise, et le maintien de l'ensemble de ses salariés liés par des contrats de travail en cours durant toute la période d'application de mesures mentionnées. En outre, l'efficacité des mesures d'accompagnement a été considérablement limitée par la lourdeur du dispositif de contrôle mis en place pour l'application de ces mesures, avec un pouvoir d'appréciation quasi souverain reconnu aux autorités administratives appelées à effectuer ce contrôle.

\section{II - L'EXÉCUTION DU TRAVAIL À L'ÉPREUVE DE LA CRISE DU COVID-19}

Dans un contexte de crise sanitaire inédite, les salariés ont essentiellement besoin d'une protection de leur intégrité physique, étant exposés au risque de contamination par le Coronavirus à l'occasion de l'accomplissement de leur activité au sein de l'entreprise. Le Code du travail a institué à la charge de l'employeur une obligation générale de sécurité, en lui imposant de prendre les mesures nécessaires et appropriées pour la prévention et la protection des salariés contre les risques professionnels. Du reste, le salarié contaminé par le Coronavirus à l'occasion de son travail est en principe pris en charge dans le cadre du régime d'assurance maladie. L'infection par le Covid-19 a été d'ailleurs assimilée à la catégorie des maladies transmissibles ${ }^{13}$.

Néanmoins, on constate l'absence de mesures spécifiques de protection sociale adaptées à l'état d'urgence engendré par le Covid-19. Ainsi, les textes adoptés par le législateur pour faire face à la crise sanitaire n'ont pas prévu d'inscrire la contamination

9 Art. 2 du décret-loi n²020-32. Voir, en application de cet article, l'arrêté du Ministre des affaires sociales en date du 20 juillet 2020 ayant fixé les secteurs et catégories d'entreprises mentionnés à cet article.

10 Arrêté conjoint du Ministre des affaires sociales et du Ministre de l'économie, des finances et de l'appui à l'investissement, en date du 25 janvier 2021.

11 La création d'un système d'assurance chômage a été envisagée dans le contrat social signé le 14 janvier 2013 entre le Gouvernement et les deux grandes organisations syndicales représentatives des travailleurs (UGTT) et des employeurs (UTICA). Mais ce système n'est toujours pas mis en place.

12 Le montant mensuel de l'indemnité a été fixé à 200 DT, c'est-à-dire l'équivalent de 61 euros.

13 Art. $1^{\text {er }}$ du décret gouvernemental n²020-152 du 13 mars 2020. 
des salariés par le Coronavirus sur la liste des maladies professionnelles ${ }^{14}$, à l'exception du cas des agents de la santé pour lesquels un arrêté du 16 novembre 2020 est venu ajouter à cette liste les infections liées au SARS-COV2 dont ils peuvent être victimes à cause ou à l'occasion de l'accomplissement de leur activité ${ }^{15}$.

En revanche, des mesures dérogatoires ont été adoptées par le législateur, introduisant une certaine flexibilité relative aux conditions du travail afin de permettre aux entreprises d'adapter ces conditions aux contraintes engendrées par les mesures de confinement général décrétées par les pouvoirs publics. À cet effet, les entreprises ont été autorisées à récupérer les heures de travail perdues suite à l'interruption de leur activité, et ce, dans un délai de 6 mois, au lieu de 2 mois, comme le prévoit l'alinéa $1^{\text {er }}$ de l'article 92 du Code du travail qui a été suspendu durant la période du confinement général ${ }^{16}$. De même, de façon dérogatoire, les entreprises ont été autorisées à accorder les congés annuels payés au titre de l'année écoulée ou de l'année en cours, sans être tenues de se conformer aux dispositions de l'alinéa $1^{\text {er }}$ de l'article 117 du Code du travail imposant d'octroyer ces congés au cours de la période du 1 er juin au 31 octobre de chaque année ${ }^{17}$.

Par ailleurs, le recours au télétravail constitue l'un des moyens les plus efficaces pour protéger les salariés contre le risque d'infection par le Coronavirus, tout en assurant la poursuite de l'activité de l'entreprise. Or, il est regrettable d'observer l'absence de mesures permettant de donner une base légale au recours au télétravail par les entreprises assujetties aux dispositions du Code du travail. En revanche, cette solution a été adoptée par le législateur s'agissant des agents publics, qui peuvent ainsi être chargés d'assurer l'exécution de leurs tâches en mode de télétravail ${ }^{18}$. Dans ce cas, les agents concernés sont tenus de se conformer à une plage horaire déterminée par le chef de l'administration ou de l'établissement.

L'inexistence d'un cadre juridique relatif au télétravail dans le secteur privé n'a pas empêché certaines entreprises de recourir à cette solution ${ }^{19}$, ce qui suppose normalement le consentement des salariés concernés. En effet, le salarié est en droit de refuser la proposition faite par l'entreprise d'exercer son travail à distance, dans la mesure où il n'existe aucune loi accordant à l'employeur le pouvoir de lui imposer une telle mesure.

Enfin, l'absence d'encadrement juridique spécifique du recours au télétravail par les entreprises assujetties aux dispositions du Code du travail atteste du caractère archaïque de ce Code, et témoigne de la nécessité d'une refonte de ses dispositions en vue d'assurer leur adaptation aux circonstances inédites liées aux crises, telles que celle qui vient de surgir avec la pandémie du Covid-19.

14 En application des dispositions de la loi n $994-28$ du 21 février 1994 portant régime de réparation des accidents du travail et des maladies professionnelles, un arrêté conjoint des Ministres de la santé et des affaires sociales en date du 10 janvier 1995 a fixé de manière limitative la liste des maladies professionnelles.

15 Arrêté du Ministre des affaires sociales et du Ministre de la santé du 16 novembre 2020, complétant l'arrêté du 10 janvier 1995 fixant la liste des maladies professionnelles.

16 Art. 3 du décret-loi du Chef du Gouvernement n²020-2 du 14 avril 2020.

17 Ibid., art. 4.

18 Art. 6 du décret-loi du Chef du Gouvernement n²020-7 du 17 avril 2020.

19 Selon l'enquête précitée sur l'impact de la crise Covid-19 sur le secteur privé (décembre 2020), réalisée par I'Institut national de la statistique, en collaboration avec International Finance Corporation, le pourcentage des entreprises ayant recouru au travail à distance a atteint $43,2 \%$ au $3^{\text {ème }}$ trimestre 2020 , contre $44,5 \%$ au $2^{\text {ème }}$ trimestre de la même année. 